\title{
Laser injection optics for high-intensity transmission in multimode fibers
}

\author{
Robert E. Setchell \\ Sandia National Laboratories, MS1421, Albuquerque, New Mexico 87185

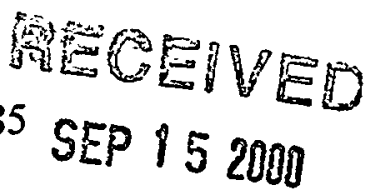 \\ ABSTRACT

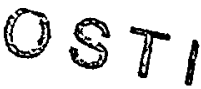

\begin{abstract}
An increasing number of applications are requiring fiber transmission of high-intensity laser pulses. Our particular interests have led us to examine carefully the fiber transmission of Q-switched pulses from multimode Nd:YAG lasers at their fundamental wavelength. The maximum pulse energy that can be transmitted through a particular fiber is limited by the onset of laser-induced breakdown and damage mechanisms. Laser breakdown at the fiber entrance face is often the first limiting process to be encountered, but other mechanisms can result in catastrophic damage at either fiber face, within the initial "entry" segment of the fiber, and at other internal sites along the fiber path. In the course of our studies we have examined a number of factors that govern the relative importance of different mechanisms, including laser characteristics, the design and alignment of injection optics, fiber end-face preparation, and fiber routing.

The present study emphasizes the important criteria for injection optics in high-intensity fiber transmission, and illustrates the opportunities that now exist for innovative designs of optics to meet these criteria. Our consideration of diffractive optics to achieve desired injection criteria began in 1993, and we have evaluated a progression of designs since that time. In the present study, two recent designs for injection optics are compared by testing a sufficient number of fibers with each design to establish statistics for the onset of laser-induced breakdown and damage. In this testing we attempted to hold constant other factors that can influence damage statistics. Both designs performed well, although one was less successful in meeting all injection criteria and consequently showed a susceptibility to a particular damage process.
\end{abstract}

Keywords: high-intensity fiber transmission, laser damage in optical fibers, beam-shaping optics

\section{INTRODUCTION}

Applications in medicine, material processing, telecommunications, and explosives technology are motivating studies of high-intensity laser transmission through optical fibers. ${ }^{1}$ As laser intensities within a fiber are increased, transmission will eventually be interrupted by laser-induced breakdown and damage processes. Our particular applications require Qswitched, multimode, Nd:YAG laser pulses at the fundamental wavelength $(1.064 \mu \mathrm{m})$ to be transmitted through stepindex, multimode, fused silica fiber. Various breakdown and damage mechanisms have been identified, as illustrated in Fig. 1. Of these processes, the first to limit fiber transmission is often a plasma-forming breakdown at the fiber entrance face. This breakdown can result in subtle surface modifications that leave the fiber face more resistant to breakdown or damage in subsequent laser pulses. Entrance-face breakdown can also be "catastrophic" in the sense that visible surface damage is produced (in the form of small pits), and subsequent laser pulses only result in greater damage. In either case, the formation of an absorptive plasma at the entrance face results in a significant reduction in the transmitted laser energy. If entrance-face breakdown does not occur during a high-intensity laser pulse, catastrophic damage can occur at the fiber exit face, within the initial "entry" segment of the fiber path, or at other internal sites. ${ }^{24}$ A smaller loss in transmitted energy typically occurs during a pulse that results in one of these damage processes.

For a given laser and fiber combination, the particular breakdown or damage mechanism that ultimately limits fiber transmission will depend on laser characteristics, the design and alignment of laser-to-fiber injection optics, fiber end-face preparation, and fiber routing. ${ }^{2}$ Entrance-face breakdown depends on the surface characteristics resulting from end-face preparation processes, and on the peak laser fluence (energy/area) at this face resulting from laser characteristics and the laser-to-fiber injection optics. The relative merits of different end-face preparation processes such as cleaving, mechanical polishing, and conditioning with a $\mathrm{CO}_{2}$ laser have been the subject of most previous investigations. A particular schedule for $\mathrm{CO}_{2}$-laser conditioning following a good mechanical polish has produced surfaces very resistant to breakdown. ${ }^{2-5}$ The other factor affecting entrance-face breakdown thresholds is the relation between the peak local fluence at this surface and 


\section{DISCLAIMER}

This report was prepared as an account of work sponsored by an agency of the United States Government. Neither the United States Government nor any agency thereof, nor any of their employees, make any warranty, express or implied, or assumes any legal liability or responsibility for the accuracy, completeness, or usefulness of any information, apparatus, product, or process disclosed, or represents that its use would not infringe privately owned rights. Reference herein to any specific commercial product, process, or service by trade name, trademark, manufacturer, or otherwise does not necessarily constitute or imply its endorsement, recommendation, or favoring by the United States Government or any agency thereof. The views and opinions of authors expressed herein do not necessarily state or reflect those of the United States Government or any agency thereof. 


\section{DISCLAIMER}

Portions of this document may be illegible in electronic image products. Images are foroduced from the best available original document. 


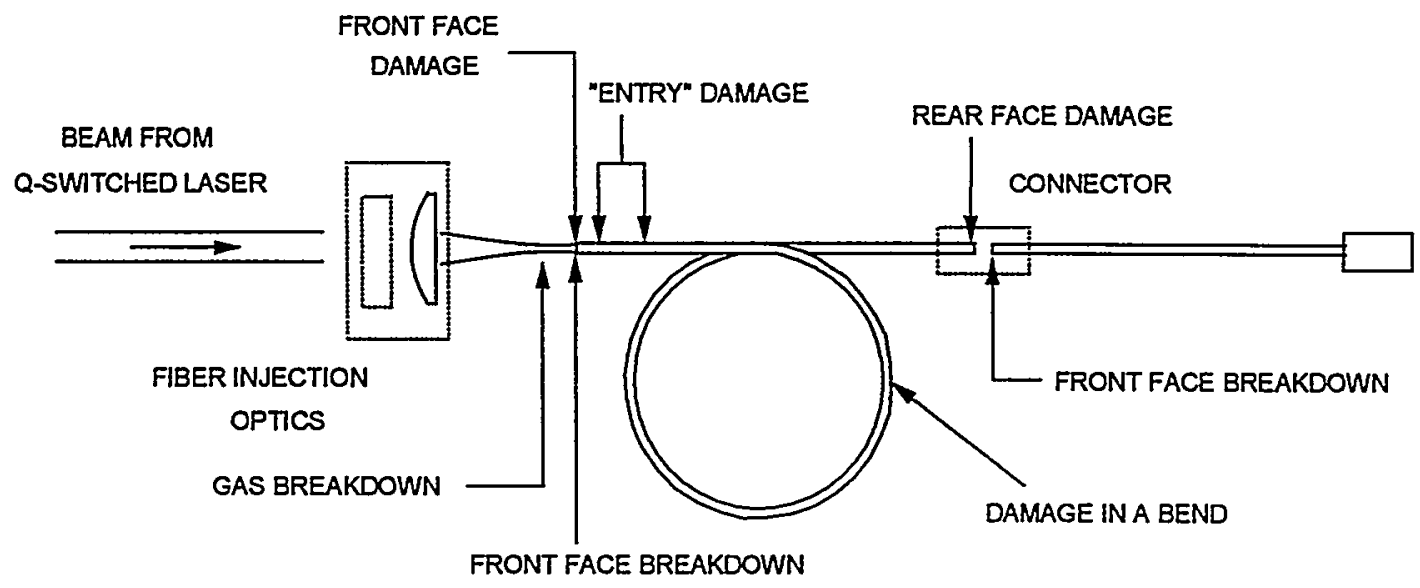

Fig. 1 Breakdown and damage processes in a high-intensity fiber transmission system

the total laser energy incident on the fiber core. By use of magnified beam-profiling techniques, the nominal fluence distribution on a fiber entrance face resulting from a particular combination of laser and injection optics can be established. A figure of merit for laser conditions at the entrance face can be defined by the following ratio of peak to average fluences:

$$
\mathrm{P} / \mathrm{A}=\frac{\text { peak local fluence }}{\text { (total energy incident on fiber core }) /(\text { fiber core area })}
$$

A perfect "flat top" fluence distribution extending over the entire core area would achieve a value of unity for this ratio. In practice, using different lasers and injection optics, we have observed values for this ratio varying from less than 3 to more than 5. The highest values have resulted from simple lens injection of multimode lasers with very strong "hot spots".

Damage beyond the entrance face but within the initially straight portion of the fiber path is denoted as "entry" damage. This damage occurs when initial reflections along the core/cladding interface result in internal beam focusing, and high local fluences are produced within one or more fiber cross sections within the "entry" region. Damage sites can appear near the core/cladding interface or nearly centered within the core, depending on the focusing pattern. Early studies ${ }^{6-8}$ found that "entry" damage was more likely if the laser axis and the initial fiber axis were not collinear. In addition, these studies found that the fiber entrance face should be positioned beyond the focal plane of the injection lens so that the beam will be diverging as it enters the fiber. A larger divergence angle, as produced by an injection lens having a shorter focal length, appeared to be desirable. In our studies, however, we have found that satisfying these conditions does not ensure that "entry" damage will be avoided. A radially expanding beam closely centered about the fiber axis can be refocused along the axis by reflections at the core/cladding interface. As will be discussed in the next section, injection optics that generate rays that do not pass through the fiber axis (skew rays) have proven effective in avoiding this particular process.

The divergence and intensity distribution of the beam entering the fiber entrance face define the initial mode power distribution within the fiber. The mode power distribution is a description of how the total laser power is distributed with respect to the angle that rays make with the fiber axis, ${ }^{9}$ with the maximum possible angle corresponding to the critical angle for total internal reflection. Unless an injection lens with a very short focal length is used, the initial mode power distribution may only extend to an angle much smaller than the maximum possible angle. In addition to enhancing "entry" damage, this condition can lead to a persistent damage mechanism within the first major bend along the fiber path. In previous studies, internal damage was often observed within a fiber segment that had transitioned from a straight entrance path to a constant-radius bend that continued through a $360^{\circ}$ loop. This damage resulted from very high fluences in the outside portion of the fiber cross section (away from the center of curvature). Although any bend in the fiber path will cause the mode power distribution to become asymmetric, the extreme asymmetry in this case was due to a significant portion of the total energy being confined to "whispering gallery" rays 10 that only reflect from the outer core/cladding boundary. To inhibit this damage process, the mode power distribution must be broadened prior to the first bend in the fiber path. A lens having an appropriately short focal length can produce rays at angles close to the maximum possible angle (that is, it will nearly fill the fiber's numerical aperture), but will likely result in very low thresholds for breakdown at 
its focal plane. In one previous study, the occurrence of damage in the initial bend was reduced by using a mechanical mode scrambler in the "entry" segment following simple lens injection. ${ }^{2}$ However, "entry" damage still occurred prior to the mode scrambler, and a new damage mechanism was introduced along the fiber path within the mode scrambler. The optimum approach for avoiding damage in an initial bend involves innovative optics that include both an injection lens with a short focal length and an element that prevents strong focusing in the focal plane of this lens.

Other damage mechanisms can be mitigated through careful routing and fixturing of fibers, and by using a careful polishing schedule that minimizes subsurface damage on fiber exit faces. ${ }^{2}$ The goals of the present study are to emphasize the important criteria for injection optics in high-intensity fiber transmission, and to illustrate the opportunities that now exist for innovative designs of optics to meet these criteria. The next section describes two recent designs for injection optics that utilize innovative elements, and identifies their properties when used with our particular test laser. This is followed by a brief description of the experimental configuration used for comparative testing of fibers with each of the designs. After descriptions of the testing and statistical fitting procedures, the results for the two designs are presented and discussed.

\section{INJECTION OPTICS}

\subsection{Criteria for injection optics and two specific designs}

As discussed in the previous section, a number of factors can be important in determining the onset of various breakdown and damage mechanisms. Injection optics are directly related to the onset of entrance face breakdown and damage, "entry" damage, and damage at an initial bend in the fiber path. An optimum design for injection optics would inhibit these processes by minimizing peak local fluences on the fiber entrance face and along the beam path prior to this face, by immediately generating a broad mode power distribution in the fiber, and by preventing refocusing patterns within the "entry" segment of the fiber path. An additional attribute would be insensitivity to small alignment errors. Considering that our applications of interest require the use of highly multimode lasers that can have strong "hot spots" in the beam and significant pulse-to-pulse variations, these design criteria are obviously very challenging.

We began developing diffractive optical elements as components for fiber injection optics in 1993. The first design was a multiple-order diffractive axicon that distributed the laser beam over a number of concentric rings on the fiber entrance face. 11 This design successfully mitigated "hot spots" and provided control of the fluence distribution over the fiber face, achieving lower values for the ratio of peak to average fluences on this face than could be achieved with simple lens injection. This element was combined with a short focal length lens, resulting in a broad mode power distribution in the fiber. The fiber entrance face was positioned at the focal plane of this lens, where peak fluences were much smaller than the fluences that would have resulted from using this lens alone. Alignment of the diffractive element with the laser beam and lens was relatively insensitive. Unfortunately, this design produced a refocusing pattern within the "entry" segment of the fiber, and catastrophic damage occurred at evenly spaced sites along the fiber axis at relatively low pulse energies. ${ }^{12}$ Subsequently, three more diffractive elements were designed, fabricated, and tested at Sandia National Laboratories for use in injection optics. These designs addressed the problem of refocusing patterns within the "entry" segment by introducing diffraction angles in planes that were perpendicular to the radial direction in these elements. When combined with the radial focusing produced by a conventional lens, skew rays that do not pass through the fiber axis are transmitted into the fiber. The last of these designs ${ }^{13}$ was successful in addressing all of the criteria for injection optics, and has been used in studies of high-intensity fiber transmission for several years. ${ }^{3,4}$

This design is also used in the current study, and a photograph is shown in Fig. 2. The diffractive element is combined with a plano-convex lens having a very short focal length $(15.2 \mathrm{~mm})$ in order to nearly fill the 0.22 numerical aperture of the fibers to be used. The element has a diameter of $6 \mathrm{~mm}$, and is composed of 87 individual segments arranged within 5 concentric rings. Each segment is a low-spatial-frequency grating that diffracts the incident light into plus and minus first and second orders. The diffraction pattern is achieved through a 14-level structure etched into a fused silica substrate. Within each segment the orientation of the grating is approximately radial, and the diffraction angles are in a plane perpendicular to this orientation. A number of skew angles are generated by having different diffaction angles at different

radial positions. The final intensity distribution at the focal plane of the lens results from the superposition of the diffaction patterns produced by each individual segment. Most of these patterns do not overlap in the focal plane, 
minimizing possible interference effects. Parameters in the design of this element were the diffraction angles, the percentage of incident light going into each order, and the geometric arrangement of the segments. These parameters permit considerable control of the fluence distributed over a fiber entrance face positioned at the focal plane of the lens. The light incident upon a particular segment appears at four locations on the fiber face, with spacings that correspond to the product of the focal length and the diffraction angles. This actively mitigates a "hot spot" in the laser near-field profile that falls on one or more of the diffractive segments. This design has resulted in our lowest measured values to date for the ratio of peak to average fluences on the fiber entrance face. As expected, the use of a lens having a very short focal length produces a broad initial mode power distribution in the fiber, and the skew angles introduced by the diffractive segments prevent refocusing patterns within the "entry" segment. In the present study this design will be referred to as a "beam scrambler."

A second promising design for injection optics combines a plano-convex lens having a short focal length (17.1 mm) with a custom beam-shaping optic consisting of an array of hexagonal lenslets. ${ }^{14}$ A photograph of a portion of this element is shown in Fig. 3. The element has an overall diameter of $8 \mathrm{~mm}$, and contains approximately 20 lenslets having an edge-to-

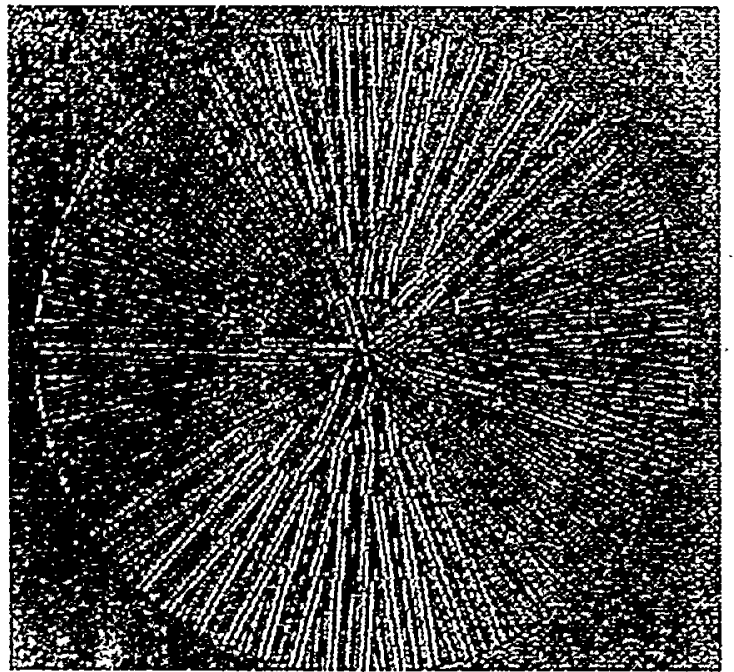

Fig. 2 Concentric rings of diffractive segments in the beam scrambler optics

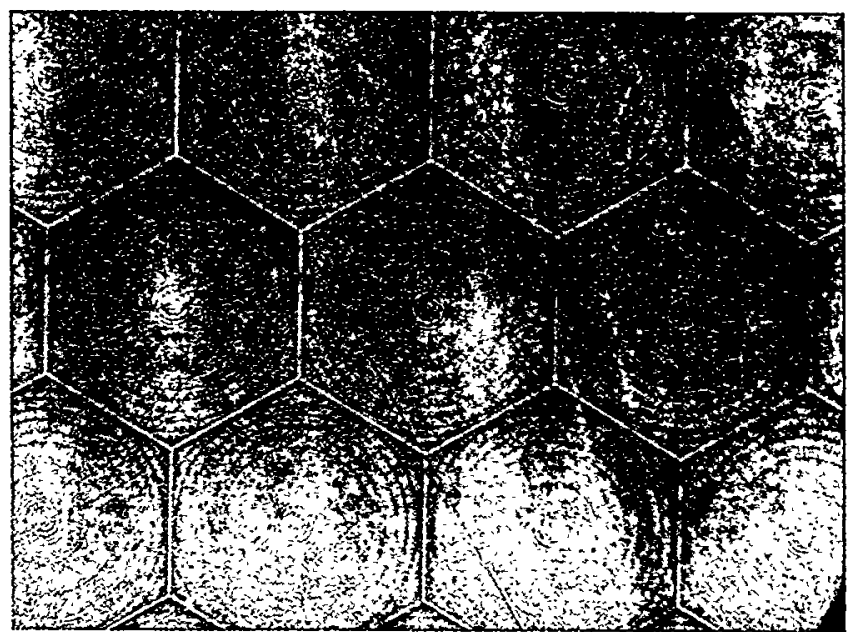

Fig. 3 Hexagonal lenslet array in the beam integrator optics

edge dimension of $1.25 \mathrm{~mm}$. The lenslets are refractive with a focal length of $69 \mathrm{~mm}$. An incident laser beam first strikes the lenslet array, where it is segmented into multiple beamlets. After passing through the primary injection lens, each beamlet passes through a focus and then forms a spot having a desired diameter at the focal plane of this lens. The spot diameter is chosen to be $85 \%$ of the core diameter of a fiber whose entrance face is positioned at this focal plane. The spots from the different beamlets overlap, and the final intensity distribution on the fiber entrance face during a laser pulse results from the superposition of these individual contributions. A brief theoretical analysis of focal plane fluences produced by this design, and a detailed experimental investigation of these fluences, have been reported. ${ }^{14}$ Diffraction effects from the finite lenslet dimensions result in a coarse modulation of the fluence profile appearing in the focal plane. Stronger, more closely spaced profile fluctuations result from interference between superimposed beamlets. The degree of interference depends on the coherence properties of the particular laser beam. For a given fiber spot size and primary lens focal length (chosen to nearly fill the fiber's numerical aperture), the remaining design parameter is the lenslet diameter (the ratio of lenslet focal length to diameter will be fixed). The lenslet diameter can be reduced in order to divide the laser beam into more overlapping beamlets, but diffraction and interference effects on the final profile will be enhanced.

The second design mitigates "hot spots" in the laser beam very effectively by dividing the cross-sectional area of the beam into smaller segments and then superimposing each of these segments over a common area filling most of the fiber core. Unless interference effects are dominant, this approach will result in lower values for the ratio of peak to average fluences on the fiber entrance face than could be achieved with simple lens injection. The previous experimental evaluation of this design showed that this ratio was quite insensitive to alignment errors. The use of a primary injection lens with a very 
short focal length will obviously produce a broad mode power distribution in the fiber. Whether or not this design is susceptible to damage due to refocusing in the "entry" segment of the fiber remained to be determined. In the present study this design will be referred to as a "beam integrator," following the nomenclature of the original authors.

\subsection{Injection properties with our test laser}

The test laser used for comparing the two designs for injection optics is an oscillator-only, multimode Nd:YAG (Laser Photonics Model YQL-102) operated at the fundamental wavelength $(1.064 \mu \mathrm{m})$ in a Q-switched, single-shot mode. This laser had been factory modified to provide a larger laser rod and flashlamp, and we had subsequently adjusted the cavity alignment to produce a more benign far-field profile. The maximum pulse energy is $200 \mathrm{~mJ}$. The average pulse width obtained in a 100-shot measurement was 9.4 ns (FWHM), with a variation of \pm 0.3 ns. Figure 4 shows the near-field profile from this laser, recorded using a CCD camera (Silicon Mountain Design Model SMD-1M15) and beam-profiling software (Spiricon Model LBA-500PC Laser Beam Analyzer). The line scans in this figure are relatively smooth, and show that the near-field profile from this multimode laser differs considerably from a "flat top" profile. Figure 5 shows this laser beam incident on the lenslet array used in the beam integrator design (recorded with a different CCD camera). As

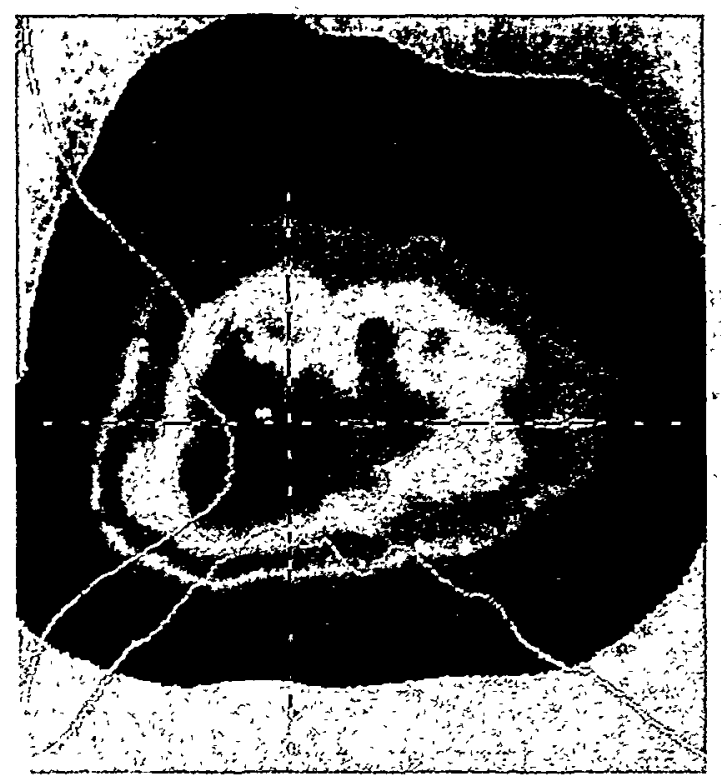

Fig. 4 Near-field profile of the test laser. Line scans extend $6.3 \mathrm{~mm}$ in width and $7.2 \mathrm{~mm}$ in height

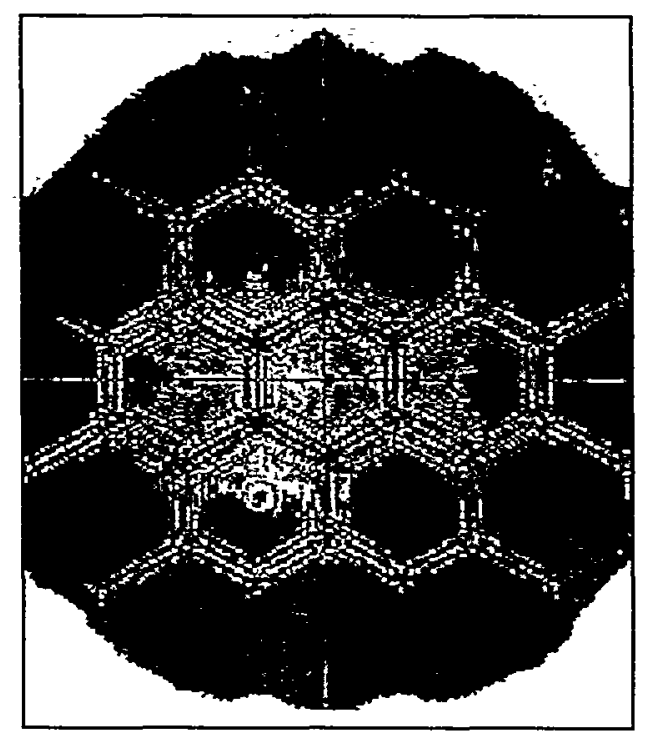

Fig. 5 Test laser incident on the lenslet array

would be expected from the profiles in Fig. 4, most of the beam energy is incident on a relatively small number of the individual lenslets. For most of these lenslets, the incident fluence is not very uniform over the lenslet area. Figure 6 shows the beam profile at the fiber entrance face obtained with our test laser and the beam integrator optics. This profile was obtained with the same equipment as Fig. 4, except that optics to provide a magnification of approximately 25 were added. The circle shown on this figure has a diameter of $365 \mu \mathrm{m}$, corresponding to the core diameter of the fibers to be tested. The beam-profiling system can provide the ratio of the peak local fluence to the average fluence within this specified diameter, and over a number of individual laser pulses this ratio was found to have an average value of 2.96 , with a variation of \pm 0.07 . As a measure of "spot size," $86.5 \%$ of the total beam energy is within a diameter of $285 \mu \mathrm{m}$.

The CCD camera used for Figs. 4 and 6 had a usable detector array of 900 by 1024 pixels, each $14 \mu \mathrm{m}$ square, with 12-bit energy resolution. To examine the effect of detector resolution on the measured ratio of peak to average fluences, we also recorded the profile at the fiber entrance face using a Cohu Model $4800 \mathrm{CCD}$ camera that had a usable detector array of 240 by 240 pixels, $23 \mu \mathrm{m}$ wide by $27 \mu \mathrm{m}$ high, with 8 -bit energy resolution. Optics providing a magnification of 10.5 were used with this camera. A comparison between horizontal line scans through the profile centroid recorded with these two cameras is shown in Fig. 7. The higher-resolution camera shows somewhat stronger and more closely spaced variations 


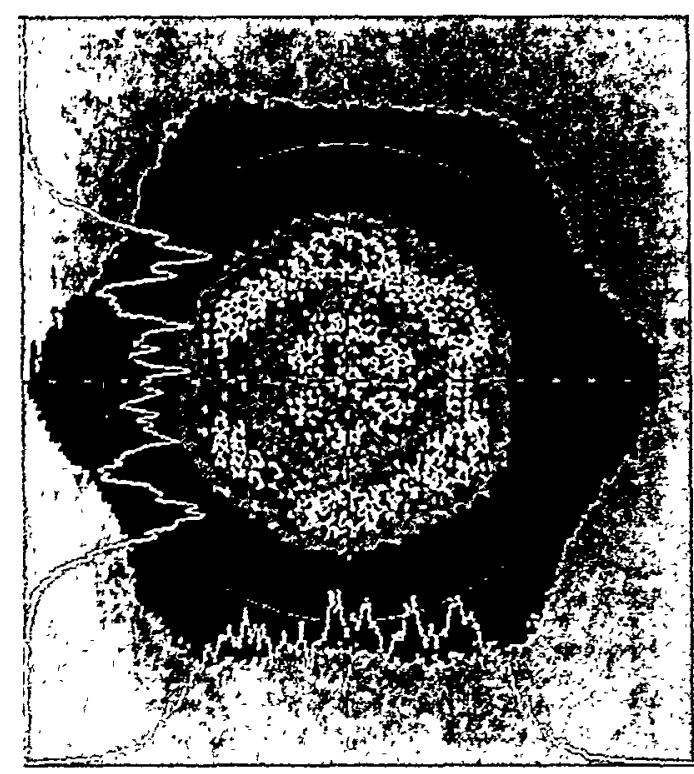

Fig. 6 Beam profile at the fiber entrance face using the beam integrator optics

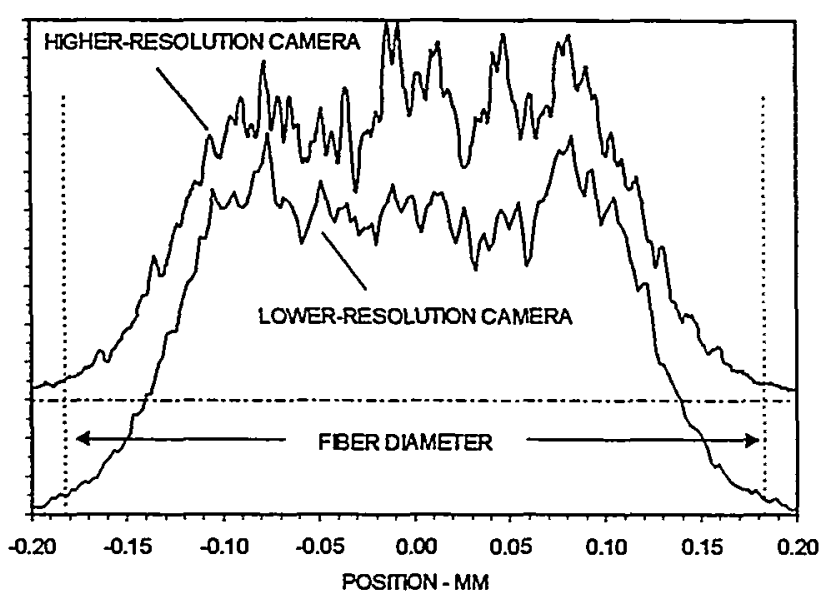

Fig. 7 Line scans of the entrance face profile (Fig. 6) using cameras with different resolutions

than obtained with the lower-resolution camera. When the lower-resolution camera was used to obtain ratios for the peak to average fluences within the fiber core area, the average measured was 2.52 , with a variation of \pm 0.08 . This significantly lower value obviously brings into question the requirements necessary for accurate measurement of fluence variations in a very small profile that must be magnified.

Figure 8 shows the beam profile at the fiber entrance face obtained with the test laser and the beam scrambler optics. A large number of spots appear distributed around concentric rings, indicating that a relatively large number of individual diffractive segments are being illuminated by the incident laser beam. Peak fluences within these spots vary considerably, however, corresponding to the fact that the "hotter" part of the near-field profile (Fig. 4) is only incident on a relatively small number of segments. This profile was obtained with the same instrumentation as Fig. 6, except that optics providing a magnification of 31 were used. The circle shown in this figure again has a diameter of $365 \mu \mathrm{m}$, and over a number of laser pulses the ratio of peak to average fluences within this diameter was found to have an average value of 2.66 , with a variation of \pm 0.07 . For this profile, $86.5 \%$ of the total beam energy is within a diameter of $320 \mu \mathrm{m}$. We also examined this profile with the lower-resolution Cohu camera, and measured an average value for the ratio of peak to average fluences of 2.20 , with a variation of \pm 0.06 . Figure 9 shows horizontal line scans taken with the two cameras. These are not scans through the profile centroid, which showed few differences, but through the innermost ring populated by diffracted spots (Fig. 8). The scan made with the higher-resolution camera again shows stronger and more closely spaced variations.

One figure of merit for injection optics is the transmission efficiency of the particular design. The plano-convex lenses used in these designs had very good anti-reflection coatings specific to the laser wavelength. For each design, a transmission efficiency can be defined in terms of the energy transmitted by a fiber with the beam integrator or beam scrambler optic in place, compared to the energy transmitted using the plano-convex lens alone (with a fixed pulse energy incident on the injection optics). The lenslet array was anti-reflection coated on both surfaces, and was found to have a transmission efficiency of $96.4 \%$ with our test laser and the fibers described in the following section. The optic with multiple diffractive segments was not anti-reflection coated on either surface, and was found to have a transmission efficiency of $76.5 \%$. If Fresnel reflection losses at both surfaces are taken into account (as if this piece had anti-reflection coatings), the transmission efficiency would have been $84 \%$. This lost energy consists of light diffracted into higher orders (which will be at angles too large to be injected into the fiber core), and light scattered from the etched silica structure.

In summary, the injection properties of the two designs when used with our test laser are similar in terms of the fluence distributions they produce on the entrance face of a test fiber. The beam scrambler design results in a larger "spot size," but produces only a slightly lower (by $\sim 10 \%$ ) ratio of peak to average fluences. Measurements made with different resolution 


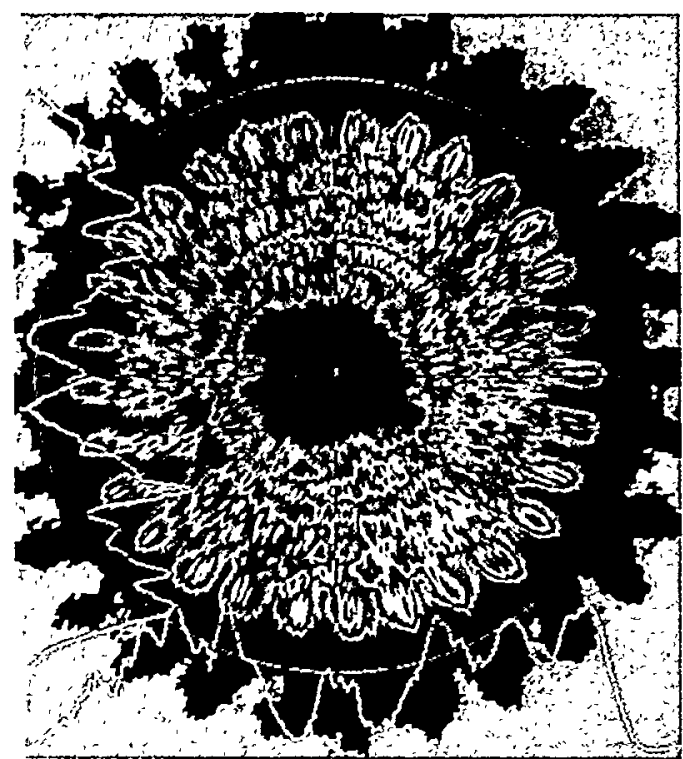

Fig. 8 Beam profile at the fiber entrance face using the beam scrambler optics

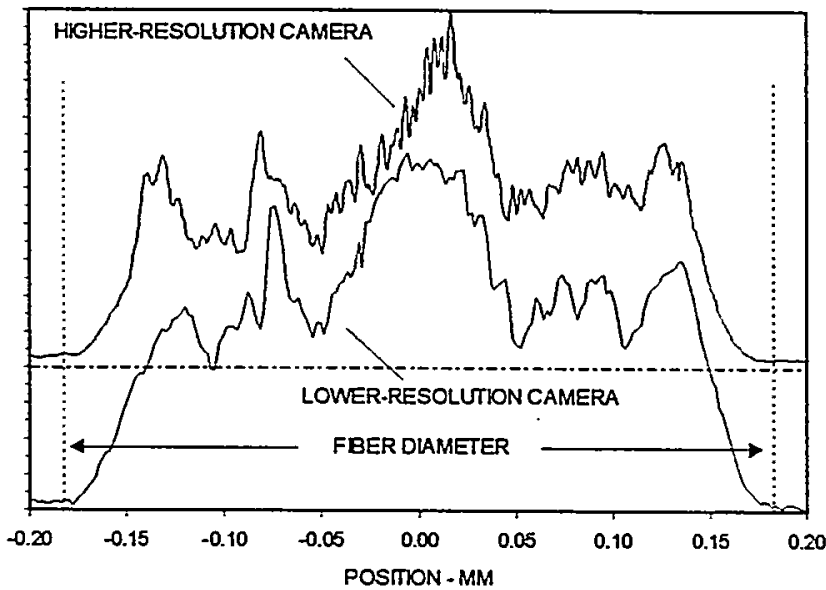

Fig. 9 Line scans of the entrance face profile (Fig. 8) using cameras with different resolutions

cameras and different optical magnifications, however, bring into question the accuracy of our absolute values for this ratio. The beam integrator design is more efficient in terms of injecting more of the incident laser energy into the fiber core.

\section{EXPERIMENTAL CONFIGURATION FOR FIBER DAMAGE TESTING}

In order to compare the performance of the two designs for injection optics, each was used to test a sufficient number of fibers to generate statistics for the onset of laser-induced breakdown and damage. We attempted to hold constant all other factors that can influence the test results so that only the injection optics would be compared. The experimental configuration used to obtain fiber damage statistics in the present study is the same as in a previous study, ${ }^{4}$ and is shown schematically in Fig. 10. The test laser was described in the previous section. The pulse energy from this linearly polarized laser is adjusted externally by means of a rotatable half-wave retardation plate followed by a polarizing beamsplitter. Discrete rotation steps of the retardation plate result in a sequence of progressively increasing pulse energies transmitted to the injection optics. For the present study an improved, regulated heat exchanger was added to the laser cooling system to obtain better pulse-to-pulse consistency over extended periods of operation. The $\mathrm{CW}$ He-Ne laser is operated collinearly with the Nd:YAG laser to enhance detection of end-face and internal damage.

The fibers used for the present testing were type FG-365-UER from 3M, having a 365- $\mu$ m diameter core of high-OH' fused silica, a 17.5- $\mu \mathrm{m}$ thick cladding of F-doped fused silica (resulting in a numerical aperture of 0.22 ), a $15-\mu \mathrm{m}$ thick TECS $^{\top M}$ coating, and a $150-\mu \mathrm{m}$ thick Tefzel ${ }^{\circledR}$ buffer. The end faces were all prepared individually according to the following schedule: ${ }^{15}$

1) Proprietary cleave by Pioneer Optics, Windsor Locks, CT

2) Buehler $3 \mu \mathrm{m}$ aluminum oxide lapping film on a metal substrate

3) Buehler $1 \mu \mathrm{m}$ aluminum oxide lapping film on a metal substrate

4) Syton HT-50 colloidal silica (South Bay Technology) on a Buehler Chemomet cloth (elastomer substrate)

Deionized water was used as the lubricant and rinse in each polishing step. This particular schedule is based on previous assessments of various end-face polishing schedules, and can produce a good breakdown-resistant surface.

\section{TEST PROCEDURE AND RESULTS}

The test procedure used for each fiber is illustrated in Fig. 11. After alignment using a very low pulse energy, the fiber was subjected to a series of single laser pulses in which the incident energy was increased with each successive pulse. Because the beam integrator design is more efficient, larger values for transmitted energy were recorded as the energy 


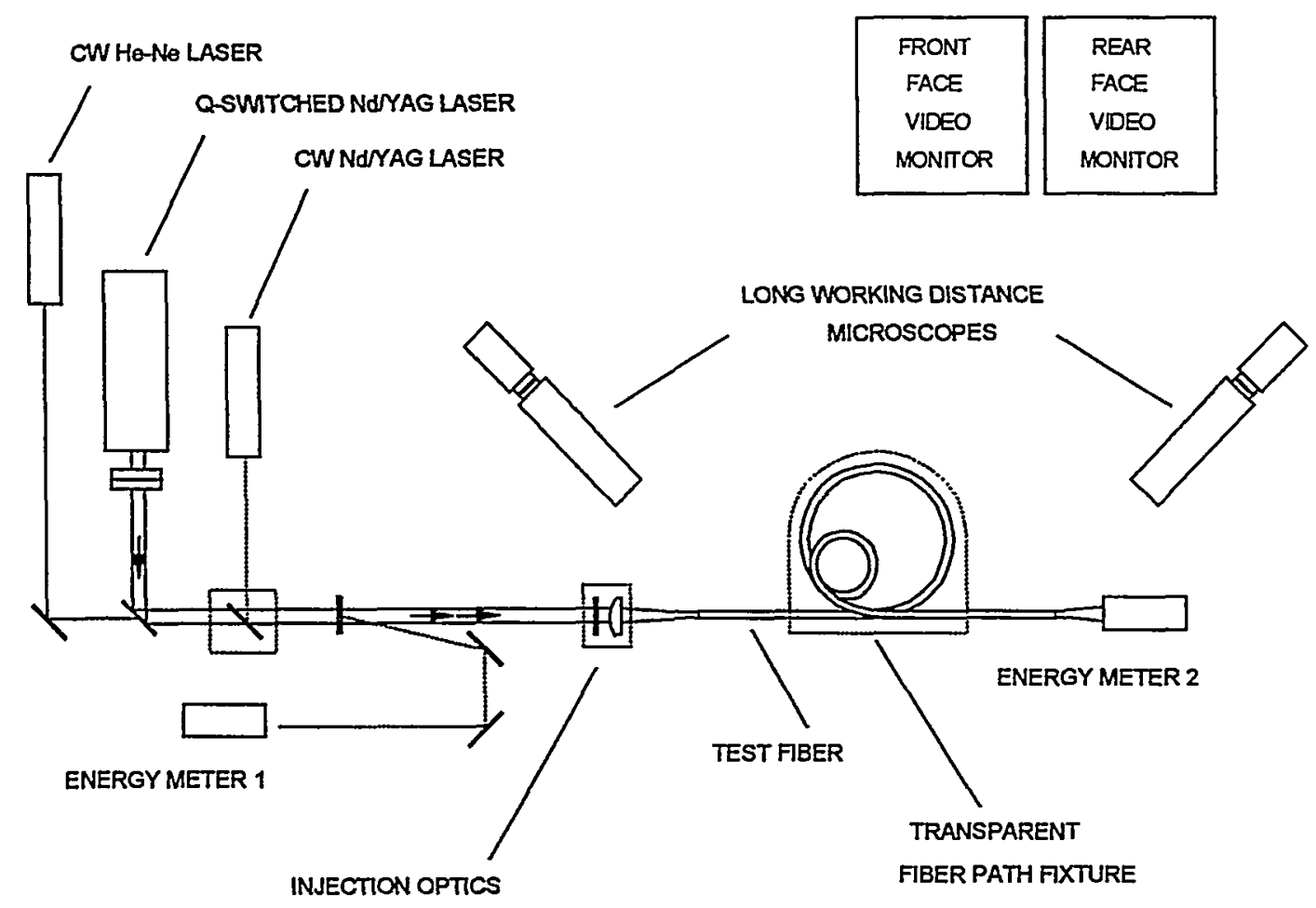

Fig. 10 Experimental configuration for fiber damage testing

incident on the injection optics was increased in fixed steps. The testing was halted when a breakdown or damage event did occur on a given shot. The maximum energy transmitted before this shot, and the attempted and actual transmitted energies during this shot, were then recorded. The collection of measured values for highest transmitted energy before breakdown or damage were then used to generate cumulative damage probability distributions. ${ }^{2}$ To represent the results in this fashion, the individual fiber data were first ordered from lowest to highest transmitted energy $E_{i}$ before damage, then assigned a corresponding rank $R_{i}$, where $R_{i}=1,2,3 \ldots . . N(N$ is the total number of fibers tested having a particular endface preparation). The cumulative probability for damage $F_{i}$ at energy $E_{i}$ was then assigned according to: ${ }^{16}$

$$
F_{i}=\left(R_{i}-0.5\right) / N
$$

A Weibull distribution in terms of the variable $E_{i}$ was obtained from a least-squares fit to the function:

$$
F(E)=1-\exp \left[-\left(E / E_{0}\right)^{m}\right]
$$

where $m$ is the Weibull slope and $E_{0}$ is a scale parameter. If a fiber did not damage by the end of the test sequence, no value of $E_{i}$ was used in the distribution fitting but the fiber was included in the total count $N$.

The transmitted energies can be readily converted to incident fluence and intensity values. When the transmitted energy reaches $100 \mathrm{~mJ}$, approximately $110 \mathrm{~mJ}$ of laser energy is incident on the fiber core. Since the core diameter is $365 \mu \mathrm{m}$, this corresponds to an average fluence of $105 \mathrm{~J} / \mathrm{cm}^{2}$ on the entrance face. Dividing by the laser pulsewidth, the average intensity on this face is then $11.2 \mathrm{GW} / \mathrm{cm}^{2}$. The peak local fluence and peak local intensity at the fiber entrance face correspond to these values multiplied by the ratio of peak to average fluences at this location, which is at least 2.96 and 2.66 for the beam integrator and beam scrambler optics, respectively.

The results of testing 20 fibers using the beam integrator optics are shown in Fig. 12. The maximum energy transmitted before breakdown or damage varied between 50 and $110 \mathrm{~mJ}$. This large variation results in a relatively small value for the Weibull slope. The transmitted energy corresponding to a $50 \%$ probability for damage was $79.2 \mathrm{~mJ}$. Two different damage mechanisms were observed during this testing. The majority of fibers experienced entrance-face breakdown, resulting in a 


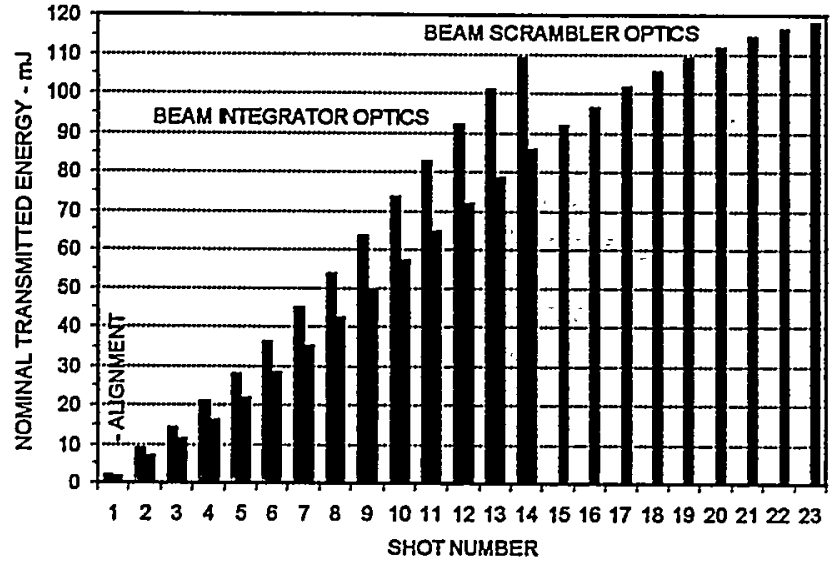

Fig. 11 Test sequences used for fiber damage testing

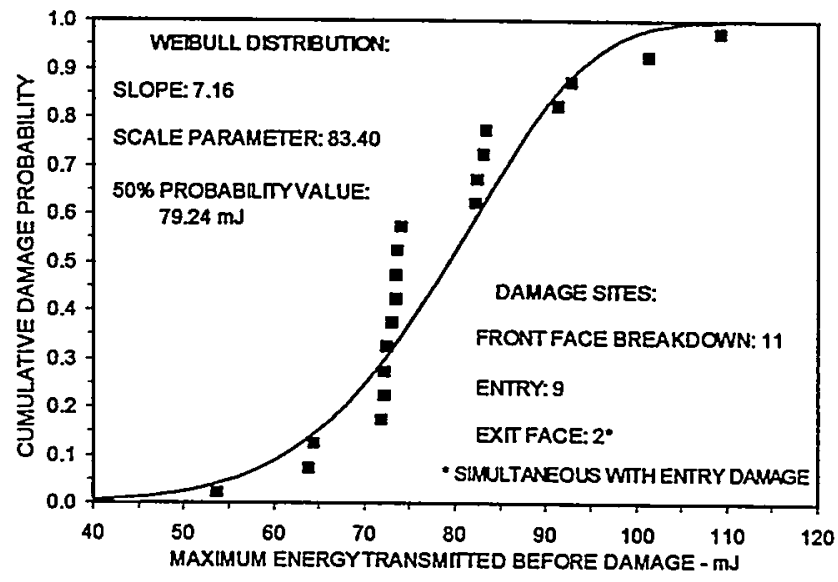

Fig. 12 Fiber testing results using the beam integrator optics

subtle damage pattern of small pits that mimicked the incident beam profile (Fig. 6). These were catastrophic damage events, as subsequent pulses would only result in stronger breakdowns and more pitting. Nine of the fibers experienced damage in the "entry" segment of the fiber path. The maximum transmitted energies in these events were uniformly scattered over the range shown in Fig. 12. The locations of these damage sites relative to the fiber entrance face are sketched in Fig. 13. These sites appeared to be roughly centered within the fiber core, rather than near the core/cladding interface. This observation, and the regular spacing of damage sites shown in Fig. 13, indicate a radial refocusing pattern occurring within the "entry" segment. A ray can be drawn entering the center of the fiber entrance face and then successively reflecting off the core/cladding interface and passing through the fiber axis at the approximate locations of all of these damage sites. This ray would enter the fiber at an angle that is consistent with the geometry of the injected beam.

The results of testing 20 fibers with the beam scrambler optics are shown in Fig. 14. The maximum energy transmitted before breakdown varied from 70 to $120 \mathrm{~mJ}$, again a fairly large range resulting in a small value for the Weibull slope. The transmitted energy corresponding to a $50 \%$ probability for damage was $92.4 \mathrm{~mJ}$. The only damage mechanism observed was entrance-face breakdown, again resulting in a subtle but catastrophic damage pattern on this face that mimicked the incident beam profile (Fig. 8). One fiber did not damage before the maximum available laser energy was reached.

\section{DISCUSSION}

In the current study, the Weibull distributions that are fitted to the testing results provide a means for comparing the two designs for injection optics, as well as for comparing with previous results. A Weibull distribution can be described by two parameters, the slope and either the scale parameter or the value for a $50 \%$ damage probability (all three were shown in Figs. 12 and 14). Weibull slopes were 7.16 and 7.40 for the beam integrator and beam scrambler, respectively, and the

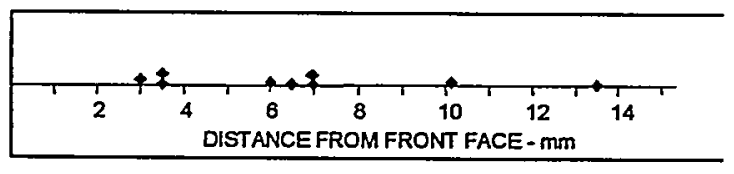

Fig. 13 Axial location of "entry" damage sites

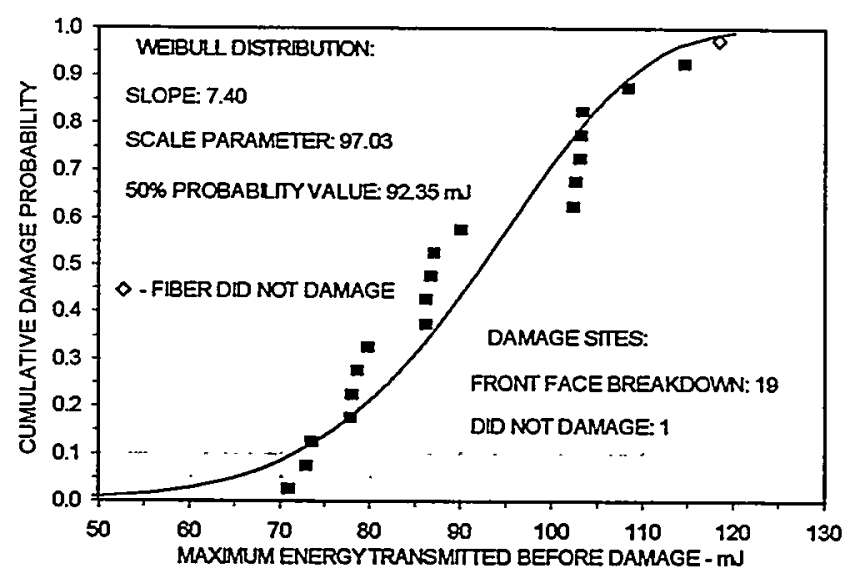

Fig. 14 Fiber testing results using the beam scrambler optics 
corresponding values for the $50 \%$ probability level were 79.2 and $92.4 \mathrm{~mJ}$. The smaller $50 \%$ probability level for the beam integrator design reflects its susceptibility to an additional damage mechanism and its slightly higher ratio of peak to average fluences at the fiber entrance face.

One consideration in comparing the present results with previous studies is that one or more factors that can affect test results were typically different in earlier studies. The test laser, beam scrambler optics, fibers, and fiber path were all the same in one earlier study, but different fiber end-face preparations were used. ${ }^{4}$ For fibers with end faces that had been $\mathrm{CO}_{2}$-laser conditioned following a good mechanical polish, a fit to the transmitted energy results gave a Weibull slope of 16.8 and a $50 \%$ probability level of $100.5 \mathrm{~mJ}$. Of the 20 fibers tested, 11 did not damage before reaching a final transmitted energy of 100-105 $\mathrm{mJ}$ (the maximum laser output was less than in the present study). The only damage mechanism observed was entrance-face breakdown. When compared to the current testing, these results demonstrate the difference between fiber entrance faces having a very good mechanical polish and faces that had been properly conditioned with a $\mathrm{CO}_{2}$ laser. The lower value for the Weibull slope in the present testing (7.40) indicates that there was more variability in the entrance-face surface characteristics in the current lot of mechanically polished fibers. In cleaning these fiber faces under a microscope, significant variations were evident in the rounding of the face edges that is typical of fibers polished in this manner. The final polishing step with colloidal silica is believed to be very important for achieving a breakdown-resistant surface, so some inadvertent fiber-to-fiber variation is this step was likely.

In another study conducted before the availability of the beam scrambler optics, simple injection with a lens having a focal length of $50 \mathrm{~mm}$ was combined with a mechanical mode scrambler in the "entry" segment. A similar test laser was used, and the fibers were the same as in the current study. ${ }^{2}$ The mode scrambler was used to broaden the mode power distribution before the first bend in the fiber path. Using fiber end faces that had been properly conditioned with a $\mathrm{CO}_{2}$ laser following a good mechanical polish, the testing results gave a Weibull slope of 8.90 and a $50 \%$ probability level of $90.1 \mathrm{~mJ}$. In comparison with more recent studies, these earlier results demonstrate the limitations to meeting fiber injection criteria with conventional optics. Most fibers damaged in the "entry" segment before the mode scrambler, within the mode scrambler, or within the initial bend in the fiber path (despite the mechanical mode mixing).

The best results we have ever obtained involved a rather impractical, "fiber-to-fiber" injection system. ${ }^{5} \mathrm{~A}$ lens with a 50 $\mathrm{mm}$ focal length was used to inject the output from a similar test laser into a length of fiber having a diameter of $600 \mu \mathrm{m}$ and a numerical aperture of 0.11 . This fiber had several $360^{\circ}$ loops in its path to mix the fiber modes. The output of this fiber was imaged with demagnification optics onto test fibers having a diameter of $400 \mu \mathrm{m}$ and a numerical aperture of 0.22. Fitting a Weibull distribution to the results obtained with $\mathrm{CO}_{2}$-laser conditioned fiber faces was not possible, as 16 out of 17 test fibers did not breakdown or damage before reaching a final transmitted energy of 100-105 mJ. This configuration came reasonably close to an ideal laser-to-fiber injection condition in which beam characteristics at the entrance face are essentially the same as would be found at an exit face after a very long fiber path.

The designs for injection optics compared in the current study obviously do not satisfy this ideal injection condition, which is probably not possible to achieve in practical applications. However, both designs offer significant improvements over what could be achieved using simple lens injection. Both designs address the important injection criteria, with the one exception being the susceptibility of the beam integrator to "entry" damage resulting from refocusing patterns. It is important to note that neither design was developed with the specific characteristics of our test laser in mind. It may be impractical to design optics based on the properties of any particular multimode laser, but it is certainly prudent to achieve the best match possible between the beam assumptions used in a design and whatever representative properties can be identified for lasers to be used in a given application. To a greater or lesser extent, both of the present designs have limitations due to diffraction and interference effects, ${ }^{14}$ and these effects need to be considered in future design efforts. With the rapid advancements now being made in design and fabrication capabilities for custom beam-shaping optics, the use of innovative injection optics should become a viable option for any application requiring high-intensity laser transmission through optical fibers.

\section{ACKNOWLEDGMENTS}

Sandia is a multiprogram laboratory operated by Sandia Corporation, a Lockheed Martin Company, for the United States Department of Energy under Contract DE-AC04-94AL85000. The author would like to thank Paul Klingsporn of 
Honeywell, Kansas City Plant, for providing the polished fiber samples used in this study. The talented assistance of Dante Berry, Sandia National Laboratories, in all phases of this work was greatly appreciated.

\section{REFERENCES}

1. J. A. Harrington, "An Overview of Power Delivery and Laser Damage in Fibers," Proc. SPIE 2966, 536 (1997). Also: the nine following papers in Proc. SPIE 2966.

2. R. E. Setchell, “An Optimized Fiber Delivery System for Q-switched, Nd:YAG Lasers," Proc. SPIE 2966, 608 (1997). Also: earlier references cited within.

3. R. E. Setchell, "End-face Preparation Methods for High-Intensity Fiber Applications," Proc. SPIE $\underline{3244}, 390$ (1998).

4. R. E. Setchell, "Effects of Accelerated Aging on Fiber Damage Thresholds," Proc. SPIE 3578, 743 (1999).

5. R. E. Setchell, "Laser-Induced Damage in Step-Index, Multimode Fibers," Proc. SPIE 1848, 15 (1993).

6. S. W. Allison, G. T. Gillies, D. W. Magnuson, and T. S. Pagano, "Pulsed Laser Damage to Optical Fibers," Appl. Opt. 24, 3140 (1985).

7. R. Pini, R. Salimbeni, and M. Vannini, "Optical Fiber Transmission of High Power Excimer Laser Radiation," Appl. Opt. $\underline{26}, 4185$ (1987).

8. W. M. Trott and K. D. Meeks, "High-Power Nd:Glass Laser Transmission Through Optical Fibers and Its Use in Acceleration of Thin Foil Targets," J. Appl. Phys. 67, 3297 (1990).

9. D. Gloge, "Optical Power Flow in Multimode Fibers," Bell Syst. Tech. J. $\underline{51}, 1767$ (1972).

10. A. W. Snyder and J. D. Love, Optical Waveguide Theory, Chapman and Hall, London, 1983, pp.180-188.

11. W. C. Sweatt and M. W. Farn, "Kinoform/Lens System for Injecting a High Power Laser Beam Into an Optical Fiber," Proc. SPIE 2114, 82 (1994).

12. R. E. Setchell, "Damage Studies in High-Power Fiber Transmission Systems," Proc. SPIE 2114, 87 (1994).

13. W. C. Sweatt, R. E. Setchell, and M. E. Warren, "Injecting a Pulsed YAG Laser Beam Into a Fiber," Proc. SPIE $\underline{3010}$, 266 (1997).

14. L. S. Weichman, F. M. Dickey, and R. N. Shagam, "Beam Shaping Element for Compact Fiber Injection Systems," Proc. SPIE 3929, 176 (2000).

15. P. Klingsporn, Honeywell, Kansas City Plant, private communication.

16. B. Bergman, "On the Estimation of the Weibull Modulus," J. Mater. Sci. Lett. 3 , 689 (1984). 